\title{
Superior Mesenteric Artery Syndrome: A Case Report of a Rare Postoperative Complication after Ileal Pouch-anal Anastomosis
}

Mohamed $\mathrm{H}^{\star}$, Mohamed SN, Omar HE and Abdelkader B

Department of Medicine and Pharmacy, Mohammed V University at Souissi, Rabat, Morocco

*Corresponding author: Mohamed H, Department of Medicine and Pharmacy, Mohammed V University at Souissi' Rabat, Morocco, Tel: 0021264756042; E-mail: mohamed.hamid@um5s.net.ma

Received Date: August 03, 2017; Accepted Date: August 23, 2017; Published Date: August 31, 2017

Copyright: (C) 2017 Mohamed H, et al. This is an open-access article distributed under the terms of the Creative Commons Attribution License, which permits unrestricted use, distribution, and reproduction in any medium, provided the original author and source are credited.

Abstract
The superior mesenteric artery syndrome following ileal pouch-anal anastomosis after total proctocolectomy is
extremely exceptional; a few cases are reported in literature. We report a new case of duodenal compression
syndrome by the superior mesenteric artery following a total proctocolectomy and ileal pouch-anal anastomosis for
familial adenomatous polyposis. The diagnosis was made four days postoperatory by a persistent vomiting and
documented by superior digestive tract occlusion showed on computed tomography scan. We attempted a medical
treatment that was efficient. The early outcome was good.

Keywords: Superior mesenteric artery syndrome; Ileal pouch-anal anastomosis; Proctocolectomy

\section{Case Report}

A 23 year old patient was diagnosed with familial adenomatous polyposis and was submitted to total proctocolectomy with manual ileal pouch-anal anastomosis by laparotomy. The ileal pouch-anal was performed covered by a protective loop ileostomy. The ileal reservoir down easily to the anal margin without any technical artifice for mesenteric elongation. The tension on the superior mesenteric artery was not considered excessive intraoperatively. On the third postoperative day the patient developed persistent vomiting and epigastric pain and had no passage of gas or liquid through the ileostomy. Perineal examination did not found any sign in favor of pelvic abscess nor pouchitis.

A CT of the abdomen revealed large gastric and first duodenal part distension with stasis shown in Figure 1, in contrast with the third part of the duodenum that describes a conical narrowing as it approached the level of the aortomesenteric constriction.

These findings supported the clinical diagnosis of superior mesenteric artery syndrome; there was no sign of ischemia in the ileal pouch. He was treated with nasogastric decompression. Drainage diminished slowly and his diet was slowly advanced. He was discharged 15 days following surgery. The early outcome was good.

\section{Comment}

The superior mesenteric artery syndrome is caused by compression of the third part of the duodenum in the angle between the aorta and the superior mesenteric artery [1,2], Frequently, predisposing medical conditions associated with catabolic states or rapid weight loss result in a decrease of the aortomesenteric angle and subsequent duodenal obstruction [1]. External compression, anatomic variants and surgical alteration of the anatomy following spine surgery or digestive surgery especially, ileoanal pouch anastomosis can also precipitate the syndrome [1,3-6]. The occurrence of prolonged postoperative ileus or small bowel obstruction after total proctocolectomy with ileo analpouch anastomosis, as seen in this case, has been reported in 6-25\% of patients $[7,8]$, but the involvement of the superior mesenteric artery syndrome was not clearly established. Following an ileo anal-pouch anastomosis, the first case is reported by Ballantyne [3] on 1987, and then, only height cases were published to date [2-4,9-11].

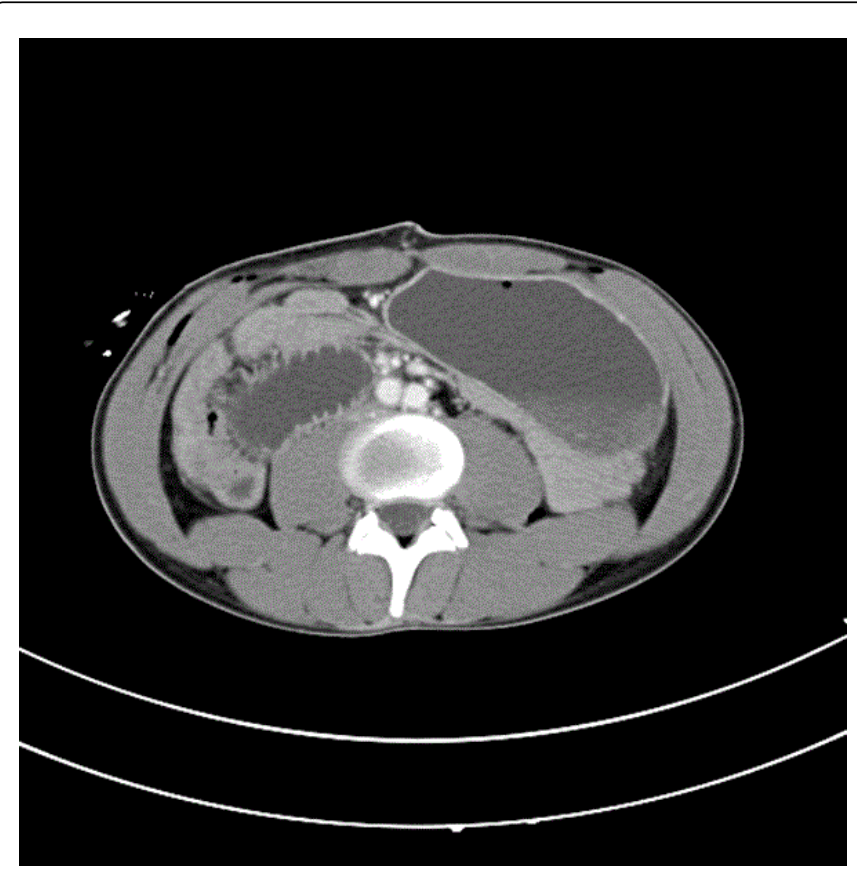

Figure 1: CT large gastric and first duodenal part distension.

The duodenum was nearly occluded between the superior mesenteric artery and aorta shown in Figures $2 \mathrm{a}$ and $2 \mathrm{~b}$. 
Citation: Mohamed H, Mohamed SN, Omar HE, Abdelkader B (2017) Superior Mesenteric Artery Syndrome: A Case Report of a Rare Postoperative Complication after lleal Pouch-anal Anastomosis. Med Rep Case Stud 2: 142. doi:10.4172/2572-5130.1000142

Page 2 of 3

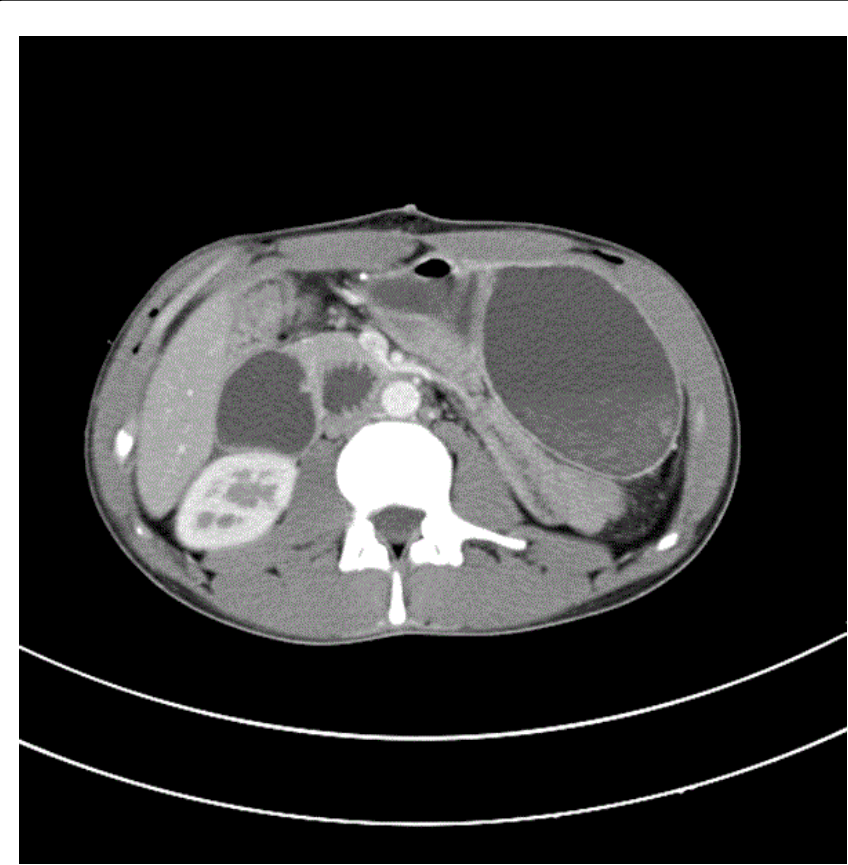

Figure 2a: The third duodenum part is occluded between the superior mesenteric artery and aorta.

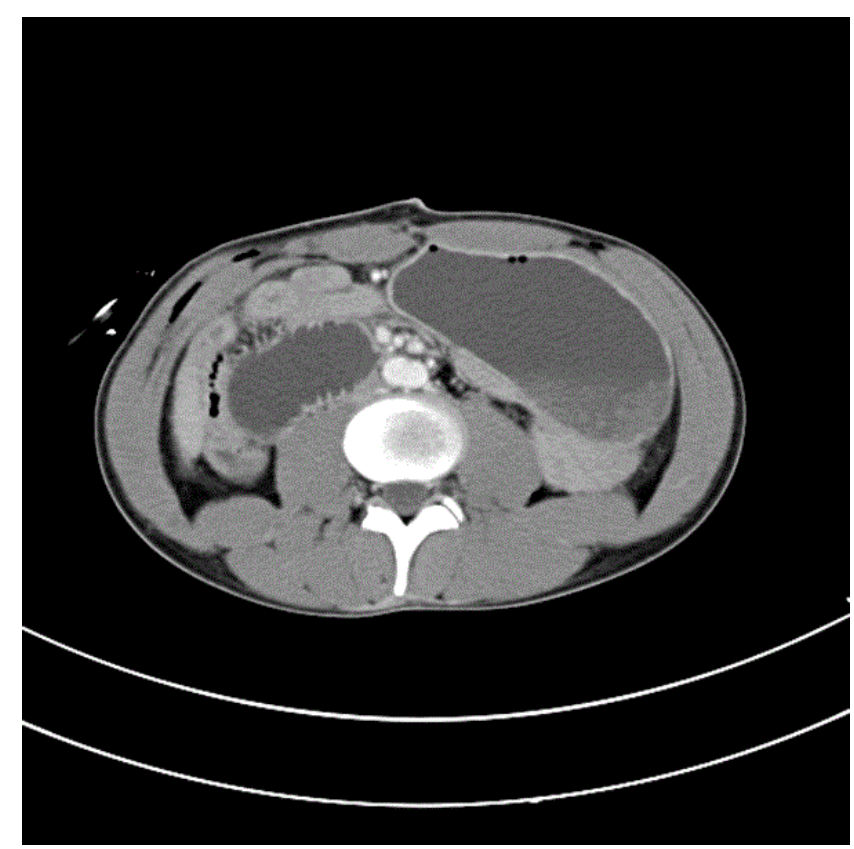

Figure 2b: The third duodenum part is occluded between the superior mesenteric artery and aorta.

The diagnosis is made by the association of vomiting, epigastric pain, and distention, with drainage of abundant gastric and bilious secretions $[1,3,9]$.

Radiological contrast examination and or computed tomography scan are considered to be appropriate for the diagnosis [2,3]. CT demonstrate the location of the obstruction that occurs between the aorta and the superior mesenteric artery as observed in the present case (Figures 1 and 2), and it also contributes to wards the diagnosis of other postoperative intestinal obstruction.

The initial treatment of this syndrome is medical. The conservative treatment consists on gastroduodenal decompression via a nasogastric tube, placing the patient in the prone or right lateral decubitus position $[2,9]$, and parenteral nutrition. In this case, the conservative treatment allowed the gastroduodenal decompression. In four cases reported, surgery was required [9]. The liberation of the duodenum from its retroperitoneal attachments, including the duodenojejunal flexure, as it was described by Strong [12] in 1958 could be more safely performed than division and reanastomosis of the duodenum [2].

In order to prevent this complication, a free tension anastomosis must be performed, during laparotomy, the top of the ileal pouch should ideally extend beyond the lower edge of the pubis by 2 to $3 \mathrm{~cm}$, and if necessary, the use of mesenteric elongation methods is recommended [13].

\section{References}

1. Welsch T, Büchler MW, Kienle P (2007) Recalling superior mesenteric artery syndrome. Dig Surg 24(3): 149-156.

2. Goes RN, Coy CS, Amaral CA, Fagundes JJ, Medeiros RR (1995) Superior mesenteric artery syndrome as a complication of ileal pouch-anal anastomosis. Report of a case. Dis Colon Rectum 38(5): 543-544.

3. Ballantyne GH, Graham SM, Hammers L, Modlin IM (1987) Superior mesenteric artery syndrome following ileal J-pouch anal anastomosis: An iatrogenic cause of early postoperative obstruction. Dis Colon Rectum 30(6): 472-474.

4. Essadel A, Benamr S, Taghy A, Lahlou MK, Mohammadine E, et al. (2001) A rare complication of ileal pouch anal anastomosis: superior mesenteric artery syndrome. Ann Chir 126(6): 565-567.

5. Boldery J, Gleeson J, Jordaan J (2006) Superior mesenteric artery syndrome following small bowel resection. ANZ J Surg 76(9): 861-862.

6. Schroeppel TJ, Chilcote WS, Lara MD, Kothari SN (2005) Superior mesenteric artery syndrome after laparoscopic Roux-en-Y gastric bypass. Surgery 137(3): 383-385.

7. Nyam DC, Brillant PT, Dozois RR, Kelly KA, Pemberton JH, et al. (1997) Ileal pouch-anal canal anastomosis for familial adenomatous polyposis: early and late results. Ann Surg 226(4): 514-519.

8. Fazio VW, Ziv Y, Church JM, Oakley JR, Lavery IC, et al. (1995) Ileal pouch-anal anastomoses complications and function in 1005 patients. Ann Surg 222(2): 120-127.

9. Matheus Cde O, Waisberg J, Zewer MH, Godoy AC (2005) Syndrome of duodenal compression by the superior mesenteric artery following restorative proctocolectomy: a case report and review of literature. Sao Paulo Med J 123(3): 151-153.

10. Ravindra KV, Rajasekhar P, Rozario AP, Pais AV, Thomas PG (1999) Superior mesenteric artery syndrome following ileo-anal pouch procedure. Indian J Gastroenterol 18(1): 35-36.

11. Tonelli F, Anastasi A, Mazzoni P, Batignani G, Monaci I, et al. (1993) The prevention of the aorto-mesenteric compression syndrome in interventions for ileoanal anastomoses. Ann Ital Chir 64(6): 675-678.

12. Strong EK (1958) Mechanics of arteriomesentric duodenal obstruction and direct surgical attack upon etiology. Ann Surg 148(5): 725-730.

13. Birnbaum DJ, Bège T, Berdah SV (2015) Surgical management of chronic inflammatory bowel disease: surgical treatment of Crohn's disease (Prise en charge chirurgicale des maladies inflammatoires chroniques de l'intestin : traitement chirurgical de la maladie de Crohn). Techniques chirurgicales - Appareil digestif 10(1): 1-13. 